\title{
Lung carcinoma therapy using epidermal growth factor receptor-targeted lipid polymeric nanoparticles co-loaded with cisplatin and doxorubicin
}

\author{
YAN NAN \\ Department of Tuberculosis, Affiliated Hospital of Hebei University, Baoding, Hebei 071000, P.R. China
}

Received February 28, 2019; Accepted August 9, 2019

DOI: $10.3892 /$ or.2019.7323

\begin{abstract}
The rate of lung cancer in tuberculosis (TB) patients is 7 to $30 \%$ higher than that in healthy individuals. Conventional chemotherapy of lung cancer shows limited efficiency due to poor tumor tissue drug accumulation and nonspecific cytotoxicity. Epidermal growth factor receptor (EGFR) is a promising target, which is overexpressed in lung carcinomas. In the present study, EGFR-targeted nanoparticles were constructed and co-delivered cisplatin (CDDP) and doxorubicin (DOX) for lung cancer therapy. In the present research, EGF-PEG-DSPE was synthesized. Then, EGFR-targeted lipid polymeric nanoparticles (LPNs) were fabricated, which consisted of a CDDP-loaded hybrophobic polymeric core, a DOX-loaded phospholipid layer, and an outer layer of EGF-PEG-DSPE ligand. The particle size, $\zeta$ potential, stability, release behavior of LPNs were characterized. The antitumor ability of LPNs were assessed in vitro and in vivo. EGFR-targeted LPNs loaded with CDDP and DOX (EGF C/D LPNs) had a size of $141.6 \mathrm{~nm}$, and could encapsulate over $80 \%$ of feed drugs. Dual drug-loaded LPNs showed synergistic effects with a combination index (CI) of 0.57. EGF C/D LPNs showed the smallest tumor volume $\left(253 \mathrm{~mm}^{3}\right)$, with a tumor inhibition ratio of $74.5 \%$. In summary, EGF C/D LPNs were stable and released the drugs in a sustained manner. In vitro and in vivo studies revealed that EGF C/D LPNs exhibited improved anticancer activity along with lower toxicity. These results indicated the best efficiency of EGF C/D LPNs for lung carcinoma therapy.
\end{abstract}

\section{Introduction}

The rate of lung cancer in tuberculosis (TB) patients is $7-30 \%$ higher than that in healthy individuals. TB also predicts

Correspondence to: Dr Yan Nan, Department of Tuberculosis, Affiliated Hospital of Hebei University, 648 Dongfeng East Road, Baoding, Hebei 071000, P.R. China

E-mail: nanyan@tom.com

Key words: lung carcinoma, epidermal growth factor receptor, combinatorial therapy, lipid polymeric nanoparticles, cisplatin, doxorubicin an increased long-term risk of cancer (1). Non-small cell lung cancer (NSCLC), the leading cause of cancer-related death, is responsible for millions of new cases and deaths every year worldwide (2). The most commonly used therapeutic methods for patients with NSCLC include surgery, radiation and systemic therapy. Systemic therapy is recommended since the consistent late diagnosis of NSCLC is a major obstacle to using surgical procedures (3-5). Cisplatin (CDDP) and doxorubicin (DOX) are commonly used as lung cancer treatment regimes in the clinic $(6,7)$. However, the toxicity and drug resistance associated with CDDP and DOX result in a high level of mortality in NSCLC (8). Thus, the delivery of anticancer agents at higher concentrations, direct targeting to the tumor site and reduced accumulation into non-tumor organs are urgently sought (8).

Epidermal growth factor receptor (EGFR) is a promising target, which is overexpressed in lung carcinomas $(9,10)$. EGFR plays an important role in regulating cell proliferation, survival and growth $(11,12)$. Many researchers have demonstrated that EGFR-targeted therapy achieves higher precision and has fewer side effects $(13,14)$. In the present study, EGFR-targeted nanoparticles were constructed and co-delivered cisplatin (CDDP) and doxorubicin (DOX) for lung cancer therapy.

Combinatorial strategies have emerged as promising therapeutic regimens to improve the anticancer efficacy, simultaneously reducing side effects $(15,16)$. Drug combinations could inhibit tumor growth through different and synergistic effect mechanisms. CDDP, a front-line DNA alkylating agent, has widely been used in solid tumors due to its various mechanisms such as DNA damage, cellular damage, mitochondria damage and dysfunction, and other deleterious effects (17-19). DOX, an anthracycline antibiotic, has antitumor cytotoxicity by interfering with DNA synthesis (20). However, the clinical application of CDDP and DOX has been severely impeded for severe toxicities, drug resistance and low aqueous solubility $(21,22)$. Current strategies to co-deliver drugs have focused on fabricating polymeric nanoparticles and lipid nanoparticles.

Lipid polymeric nanoparticles (LPNs) combine the merits of both polymeric nanoparticles and lipid nanoparticles. Therefore, LPNs have been demonstrated to display distinctive features in combinational therapy such as high biocompatibility, high drug loading and in vivo stability, low cytotoxicity, controlled release and capability for modifications 
and conjugations (23). In the present study, EGF-PEG-DSPE was synthesized. Then, EGFR-targeted LPNs were fabricated, which consisted of a CDDP-loaded hybrophobic polymeric core, a DOX-loaded phospholipid layer, and an outer layer of EGF-PEG-DSPE ligand. The particle size, $\zeta$ potential, stability, and release behavior of the LPNs were characterized. The antitumor ability of LPNs was assessed in vitro and in vivo.

\section{Materials and methods}

Materials. Murine EGF was purchased from PeproTech (Rocky Hill, NJ, USA). CDDP injection was provided by Hanson Pharma Co., Ltd. (Lianyungang, China). CDDP, DOX, PLA, fetal bovine serum (FBS), Roswell Park Memorial Institute (RPMI)-1640 medium and 3-(4,5-dimethyl-2-thiazolyl)2,5-diphenyl-2-H-tetrazolium bromide (MTT) were purchased from Sigma-Aldrich; Merck KGaA. Miglyol ${ }^{\circledR} 812$ was provided by Beijing Fengli Jingqiu Pharmaceutical Co., Ltd. (Beijing, China). DSPE-PEG ${ }_{2000}$-maleimide was purchased from Peng Sheng Biological (Shanghai, China).

Cell line and culture. A549 cells were obtained from the American Type Culture Collection (ATCC; Manassas, VA, USA). Cells were maintained in RPMI-1640 medium supplemented with $10 \%$ (v/v) FBS, $50 \mu \mathrm{M}$ 2-mercaptoethanol, and $100 \mu \mathrm{g} / \mathrm{ml}$ kanamycin at $37^{\circ} \mathrm{C}$ in a humidified atmosphere of $5 \% \mathrm{CO}_{2}$.

Animal model. Male C57BL/6 mice (6 weeks of age, 20-25 g) were purchased from Beijing Vital River Laboratory Animal Technology Co., Ltd. (Beijing, China) and raised under conventional conditions with a 12-h light/dark cycle, constant temperature $\left(25^{\circ} \mathrm{C}\right)$ and humidity $(60 \%)$, and free access to standard food and water (24). To produce the animal model, the mice were injected with A549 cells $\left(1 \times 10^{6}\right.$ cells in $100 \mu \mathrm{l}$ PBS/mouse) into the right flank, followed by assays for tumor growth. Tumor volume (TV) was determined by the formula: (Largest superficial diameter) x (Smallest superficial diameter $)^{2} / 2$. When the tumor diameter reached $12 \mathrm{~mm}$, the body weight lost was over $25 \%$, or the mice were too weak to take food and water, they were sacrificed. Eighty mice were used and euthanized; no mouse was found dead. Animal health and behavior were monitored every $12 \mathrm{~h}$ along with the tumor growth. When any animal experienced mild pain (shortly arched back, especially after administration; partly hair rising; occasional salivation; and transient tremor) during the experiments, oxymorphone $(0.05 \mathrm{mg} / \mathrm{kg}$, s.c.) was administrated. Barbital sodium was administrated $(100 \mathrm{mg} / \mathrm{kg}$, i.p. injection) to the mice and then mice were sacrificed by cervical dislocation. All animal experiments were approved by the Ethics Committee of the Affiliated Hospital of Hebei University and followed the SUNY Upstate Medical University, Department of Laboratory Animal Resources Guidelines for Anesthesia and Analgesia in Laboratory Animals and Guidelines for the Care and Use of Laboratory Animals and the National Animal Laboratory Center of China (http://www.cmu.edu.cn/sydwb/info/1841/1211.htm).

Synthesis and characterization of EGF-PEG-DSPE. EGF was modified with an excess of Traut's reagent for $1 \mathrm{~h}$ at room temperature under nitrogen to obtain thiolated EGF (Fig. 1A) (25). Excess thiolated EGF was mixed with DSPE-PEG $_{2000}$-maleimide and stirred at $400 \mathrm{rpm}$ at room temperature overnight. To remove unbound EGF, the sample was separated by gel filtration with Sephadex G-150 gel and eluted with HEPES buffer in $600 \mu \mathrm{l}$ fractions. ${ }^{1} \mathrm{H}-\mathrm{NMR}$ (DMSO-d6, $300 \mathrm{MHz}$ ) spectroscopy was used for the confirmation of the chemical structure (26).

Preparation of EGFR-targeted LPNs. EGFR-targeted LPNs loaded with CDDP and DOX (EGF C/D LPNs, Fig. 2) were prepared by a solvent extraction/evaporation method (27). Organic phase was formed by dissolving CDDP (100 mg) and PLA $(200 \mathrm{mg})$ in dichloromethane $(5 \mathrm{ml})$. An aqueous phase was obtained by dispersing DOX (50 mg), Miglyol ${ }^{\circledR} 812$ $(100 \mathrm{mg})$ and EGF-PEG-DSPE $(100 \mathrm{mg})$ in water $(20 \mathrm{ml})$ by ultrasonication. The organic solution phase was then added into the aqueous phase and ultrasonicated for $5 \mathrm{~min}$ in an ice bath. The organic solvent was then removed by stirring at the speed of $300 \mathrm{rpm}$ with a magnetic stirrer.

EGFR-targeted LPNs loaded with CDDP (EGF C LPNs) were prepared using the same method without adding DOX.

EGFR-targeted LPNs loaded with DOX (EGF D LPNs) were constructed in a similar manner without adding CDDP.

Drug-free EGFR-targeted LPNs (EGF LPNs) were constructed in a similar manner without adding any drug.

EGFR-free LPNs loaded with CDDP and DOX (C/D LPNs) were constructed in a similar manner using PEG-DSPE instead of EGF-PEG-DSPE.

Free-CDDP and DOX drug combination (Free C/D) was constructed by dissolving DOX (25 mg) in CDDP injection (10 ml, $5 \mathrm{mg} / \mathrm{ml})$.

Characterization of LPNs. The size, polydispersity index (PDI), and $\zeta$ potential of the LPNs were measured by a Malvern Zetasizer Nano ZS90 (Malvern Instruments, Malvern, UK) (28). A UV-vis spectrophotometric method was used to measure the drug encapsulation efficiency (EE) and drug loading (DL) capacity of CDDP or DOX in LPNs (29). LPNs were dissolved in o-phenylenediamine and dimethylformamide (DMF) and heated at $90^{\circ} \mathrm{C}$ for $30 \mathrm{~min}$. DMF-water mixture (7:3, v/v, $\mathrm{pH} 6.2)$ was used to dilute the product and estimated at $478 \mathrm{~nm}$ (for DOX) and $705 \mathrm{~nm}$ (for CDDP) on the UV-vis spectrophotometer (Simadzu, Japan) (30). The EE and DL were determined according to the formulas: $\mathrm{EE}(\%)=\left(\right.$ weight $_{\text {entapped drug }} /$ weight $\left.{ }_{\text {total drug }}\right) \times 100$; DL $(\%)=\left(\right.$ weight $_{\text {entapped drug }} /$ weight $\left._{\text {LPNs }}\right) \times 100$.

Stability of LPNs. LPNs were stored at $2-8^{\circ} \mathrm{C}$. The size and EE of the LPNs were measured for 3 month to determine the storage stability (8). At 0, 5, 10, 20, 30, 60, and 90 days, LPNs were taken out and assessed by the methods mentioned in 'Characterization of LPNs' section.

Drug release from LPNs. Drug release from LPNs was evaluated by a dialysis method (31). Various types of LPNs $(2 \mathrm{ml})$ were sealed in dialysis bags (molecular cut-off: $1 \mathrm{kDa}$ )

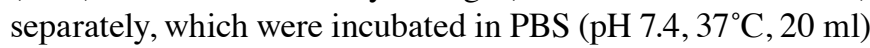
and shaken at a speed of $100 \mathrm{rpm}$. Incubation medium was collected and replaced by the same volume of fresh pre-heated 
A<smiles>N=C1CCCS1</smiles><smiles>NC(Cl)(CCC[SH2+])NC(=O)[O-]</smiles><smiles>CCCCCCCCCCCCCCCCCCCCCCCCCCCCC(=O)CCCOC(=O)CCCCCCC</smiles>

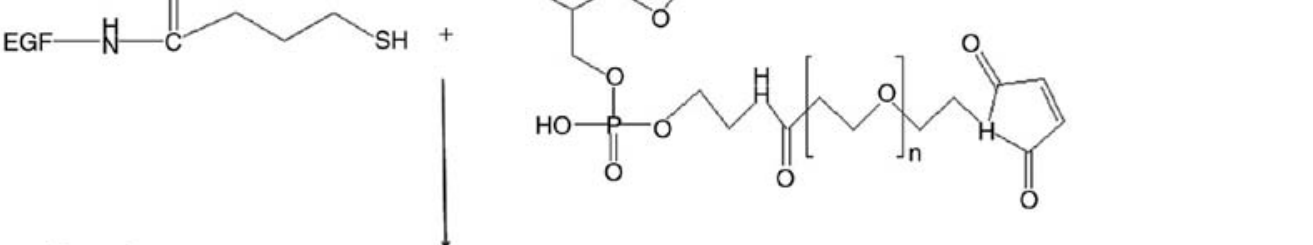

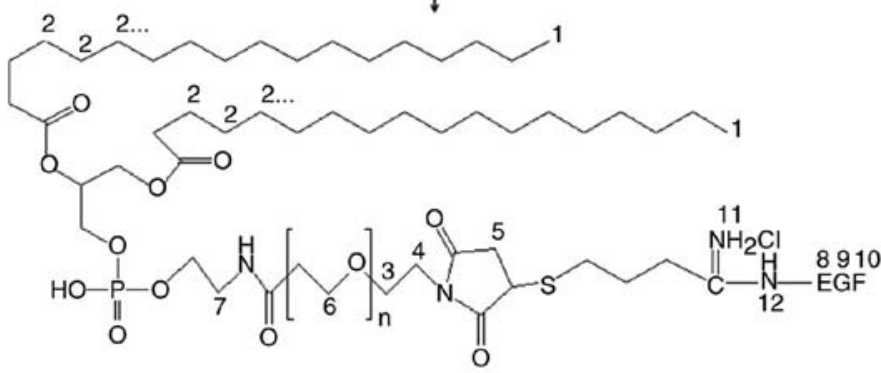

B

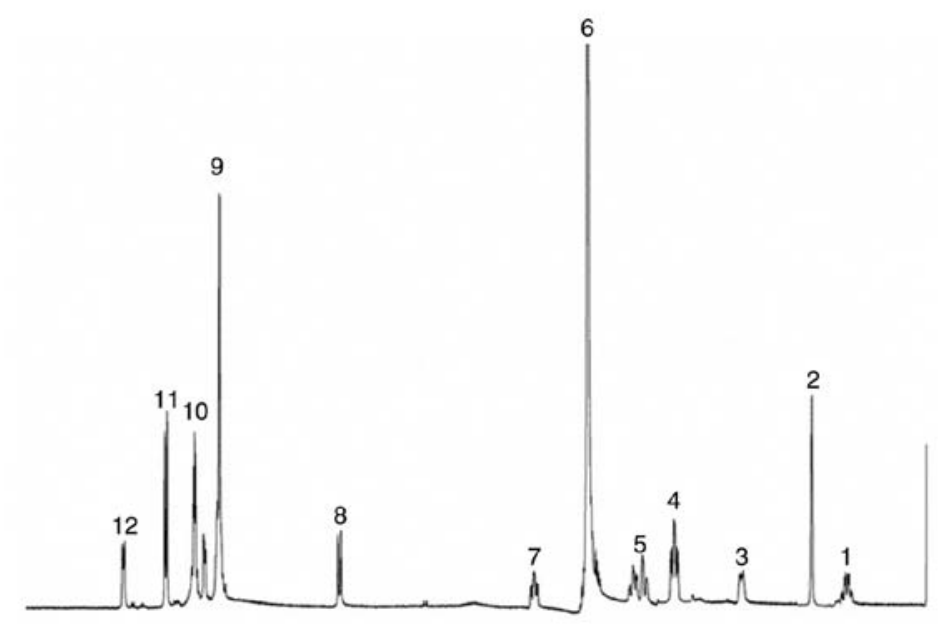

$\begin{array}{llllllllllllllllllll}8.5 & 8.0 & 7.5 & 7.0 & 6.5 & 6.0 & 5.5 & 5.0 & 4.5 & 4.0 & 3.5 & 3.0 & 2.5 & 2.0 & 1.5 & 1.0 & \mathrm{ppm}\end{array}$

Figure 1. Synthesis route (A) and ${ }^{1} \mathrm{H}-\mathrm{NMR}$ spectroscopy (B) of EGF-PEG-DSPE. EGF was modified with an excess of Traut's reagent for $1 \mathrm{~h}$ at room temperature under nitrogen to obtain thiolated EGF. Excess thiolated EGF was mixed with DSPE-PEG ${ }_{2000}$-maleimide and stirred at $400 \mathrm{rpm}$ at room temperature overnight. ${ }^{1} \mathrm{H}-\mathrm{NMR}$ spectroscopy indicates the chemical structure shifts marked with numbers according to the structure. EGF, epidermal growth factor.

medium at specific time points. The medium containing the released drugs was analyzed by the methods described in the above 'Characterization of LPNs' section.

Cytotoxicity of LPNs. Cytotoxicity of the LPNs was evaluated on A549 cells by MTT assay (32). Cells ( $2 \times 10^{5}$ per well) were seeded into 6-well microplates and allowed to grow for $24 \mathrm{~h}$ to a subconfluent state. The various types of LPNs and Free C/D were added along with fresh medium (contained $10 \%$ of FBS) and incubated for $72 \mathrm{~h}$. MTT solution (50 $\mu \mathrm{l}$ in culture medium) was then added after suspensions were removed . Thereafter, the cells were incubated at $37^{\circ} \mathrm{C}$ in $5 \% \mathrm{CO}_{2}$ for another $4 \mathrm{~h}$ and the medium was removed. DMSO $(100 \mu 1)$ was then added to dissolve the crystals and the absorbance was measured using a microplate reader at $570 \mathrm{~nm}$. The formula: $\left(\right.$ Absorbance $\left._{\text {test cells }}\right) /\left(\right.$ Absorbance $\left._{\text {control }}\right)$ x 100 was used to calculate the viability of the treated cells.
Synergistic effects of LPNs. Combination index (CI) analysis was undertaken to study the synergistic effect of the CDDP and DOX combination formulation (8). A CI value $>1$ indicates an antagonistic effect, $<1$ indicates a synergistic effect. To evaluate the $\mathrm{CI}$ value, $\mathrm{IC}_{50}$ values of the various types of LPNs described in the above section were calculated. CI values were calculated according to the following formula: $\mathrm{CI}_{50}=\mathrm{D}_{\mathrm{CDDP}} /\left(\mathrm{D}_{50}\right)_{\mathrm{CDDP}}+\mathrm{D}_{\mathrm{DOX}} /\left(\mathrm{D}_{50}\right)_{\mathrm{DOX}} \cdot \mathrm{D}_{\mathrm{CDDP}}$ and $\mathrm{D}_{\mathrm{DOX}}$ mean the $\mathrm{IC}_{50}$ value of CDDP and DOX, separately. $\left(\mathrm{D}_{50}\right)_{\mathrm{CDDP}}$ and $\left(\mathrm{D}_{50}\right)_{\text {DOX }}$ referred to the concentrations of CDDP and DOX in the $\mathrm{LPN}$ formulations at the $\mathrm{IC}_{50}$ value.

In vivo tissue distribution of LPNs. The mice bearing the lung carcinoma model were randomly divided into several groups and intravenously administered (through the tail vein) $200 \mu \mathrm{l}$ of the various types of LPNs and Free C/D (each contained $5 \mathrm{mg}$ CDDP per $\mathrm{kg}$ and/or $2.5 \mathrm{mg}$ DOX per $\mathrm{kg}$ of the mice), 


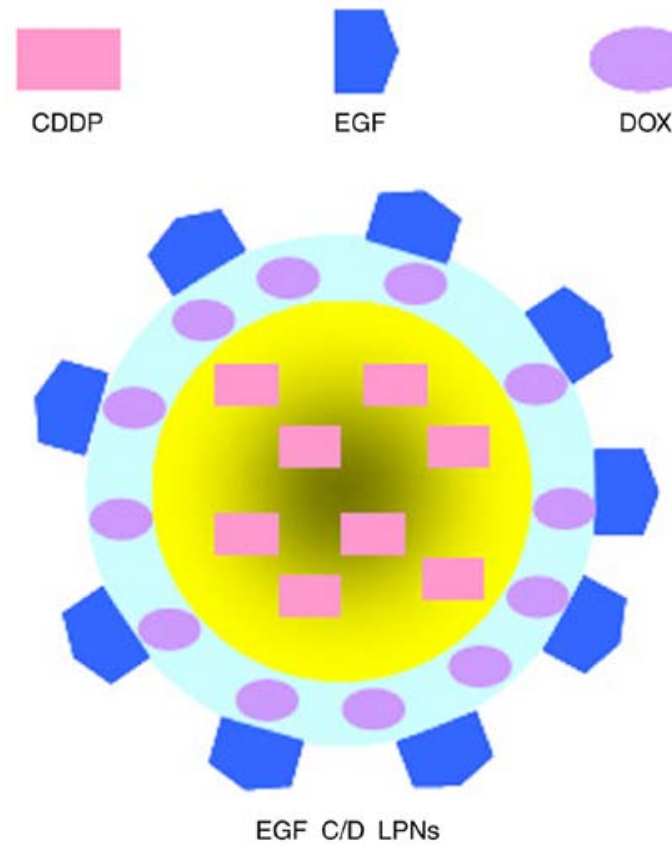

Figure 2. Schematic illustration of the EGF C/D LPNs. EGF C/D LPNs were prepared by a solvent extraction/evaporation method. EGF C/D LPNs, EGFR-targeted LPNs loaded with CDDP and DOX. CDDP, cisplatin; DOX, doxorubicin; EGF, epidermal growth factor; LPNs, lipid polymeric nanoparticles.

separately (33). At 1 and $24 \mathrm{~h}$ after injection, the mice were sacrificed and the normal and tumor tissues of the mice were collected, using $0.9 \%$ saline solution to homogenize. The tissue content of CDDP and DOX was determined as described in the above 'Characterization of LPNs' section.

In vivo antitumor efficiency of LPNs. The same amount of drugs were administered to lung carcinoma-bearing mice using the same method every three days, respectively (32). Mice injected with $0.9 \%$ saline were used as control. The tumor growth and body weight changes of each mice were measured every three days. At day 18 after first drug administration, mice were sacrificed by cervical dislocation and the tumors were excised and weighed. The formula: (Tumor weight $_{\text {control-tumor weight }}$ treated $_{\text {t }} /\left(\right.$ tumor weight $\left._{\text {control }}\right) \times 100$ was applied to calculate the tumor inhibition ratio (\%).

Statistical analysis. The study data are expressed as the mean \pm standard deviation. Statistical analysis was performed using ANOVA followed by a post hoc test (S-N-K method). A P-value $<0.05\left({ }^{*} \mathrm{P}<0.05\right)$ was considered to indicate a statistically significant result.

\section{Results}

Characterization of EGF-PEG-DSPE. ${ }^{1} \mathrm{H}-\mathrm{NMR}$ spectroscopy was utilized to determine the chemical structure of EGF-PEG-DSPE. Fig. 1B presents the chemical structure shifts and are marked with numbers according to the structure, ঠ: (1) $0.79\left(\mathrm{CH}_{3}\right.$, DSPE); (2) $1.18\left(\mathrm{CH}_{2}, \mathrm{DSPE}\right)$; (3) $1.89\left(\mathrm{CH}_{2}\right.$, beside the $\mathrm{O}$ side of PEG); (4) $2.47\left(\mathrm{CH}_{2}\right.$, beside the $\mathrm{N}$ side of thiolated EGF); (5) 2.89 ( $\mathrm{CH}_{2}$, thiolated EGF); (6) 3.37
$\left(\mathrm{CH}_{2}, \mathrm{PEG}\right)$; (7) $3.88\left(\mathrm{CH}_{2}\right.$, beside the $\mathrm{NH}$ side of amide linkage); (8-10) 5.86-7.37 $\left(\mathrm{CH}_{2}, \mathrm{EGF}\right) ;(11) 7.57\left(=\mathrm{NH}_{2} \mathrm{Cl}\right)$; (12) 8.03 (-NH-EGF).

Characterization of LPNs. Table I shows the physicochemical property of LPNs. When EGF was added to the formulation, particle size was increased from $118.7 \mathrm{~nm}$ (C/D LPNs) to $141.6 \mathrm{~nm}$ (EGF C/D LPNs). However, the size did not increase with the feeding of drugs; EGF LPNs and EGF C/D LPNs had similar sizes $(\sim 140 \mathrm{~nm})$. The PDIs of LPNs were between 0.10 and 0.20 . Negative $\zeta$ potential was achieved by the LPNs and LPNs containing EGF were more negatively charged. More than $80 \%$ (EE) of the drugs were loaded into the LPNs, with various DL capacities from 2.2 to $4.7 \%$. The stability of LPNs was then evaluated over the period of 90 days. Fig. 3 shows that the size and EE of LPNs did not change significantly.

Drug release from LPNs. In vitro CDDP and DOX release profile of various types of LPNs all showed sustained behaviors (Fig. 4). However, the release rates of DOX were faster than CDDP, which may be explained by the drugs loaded in the different parts of LPNs. In addition, LPNs containing EGFR released drugs more slowly than the EGFR-free LPNs.

Synergistic effects of LPNs. Table II summarized the $\mathrm{CI}_{50}$ of EGF C/D LPNs on A549 cells when different CDDP/DOX ratios were applied. Synergistic effect $\left(\mathrm{CI}_{50}=0.57\right)$ was observed at the ratio of 2:1 (CDDP/DOX, w/w), suggesting the suitable drug ratios for the LPN preparation. Other weight ratios tested all showed $\mathrm{CI}_{50}$ values $>1$, which indicated antagonistic or no obvious synergistic effect.

Cytotoxicity of LPNs. Fig. 5 shows that there was no significant cytotoxicity of the drug-free EGF LPNs. This indicates the safety of the materials used in the studied concentrations. Drug-containing formulations showed cytotoxicity in dose-dependent manners. EGF C/D LPNs showed markedly higher inhibition efficiency in lung carcinoma cells compared with C/D LPNs, EGF C LPNs and EGF D LPNs $(\mathrm{P}<0.05)$. $\mathrm{C} / \mathrm{D}$ LPNs showed more efficiency than Free $\mathrm{C} / \mathrm{D}(\mathrm{P}<0.05)$.

In vivo tissue distribution of $L P N s$. Fig. 6 shows the in vivo drug tissue distribution of EGF C/D LPNs, C/D LPNs and Free C/D in the lung carcinoma mouse model. At $24 \mathrm{~h}$ after injection, the CDDP and DOX tumor distribution of EGF C/D LPNs was higher in the tumor tissue than that of the C/D LPNs and Free $\mathrm{C} / \mathrm{D}(\mathrm{P}<0.05)$. CDDP and DOX containing LPNs accumulated less in the heart and kidney than Free C/D at $1 \mathrm{~h}$ after injection $(\mathrm{P}<0.05)$.

In vivo antitumor efficiency of LPNs. The tumor volume curves illustrated that treatment with drug-free EGF LPNs did not show any effect on suppressing tumor growth when compared with the $0.9 \%$ saline control (Fig. 7A). However, when loaded with drugs, LPNs showed profound tumor growth inhibition ability, compared with Free $\mathrm{C} / \mathrm{D}$ group $(\mathrm{P}<0.05)$. The tumor volume following treatment with EGF C/D LPNs at day 18 was $253 \mathrm{~mm}^{3}$, which was the smallest among all the tested groups. The larger tumor size following treatment with C/D LPNs $\left(512 \mathrm{~mm}^{3}\right)$ compared with EGF C/D LPNs at the 
Table I. Physicochemical properties of the LPNs.

\begin{tabular}{|c|c|c|c|c|c|}
\hline Characteristics & EGF C/D LPNs & EGF C LPNs & EGF D LPNs & EGF LPNs & C/D LPNs \\
\hline Size (nm) & $141.6 \pm 4.6$ & $139.8 \pm 4.3$ & $143.1 \pm 4.9$ & $140.2 \pm 3.9$ & $118.7 \pm 3.5$ \\
\hline PDI & $0.19 \pm 0.02$ & $0.17 \pm 0.02$ & $0.18 \pm 0.03$ & $0.15 \pm 0.01$ & $0.13 \pm 0.01$ \\
\hline$\zeta$ potential $(\mathrm{mV})$ & $-39.2 \pm 3.7$ & $-36.7 \pm 3.5$ & $-38.1 \pm 3.1$ & $-37.3 \pm 2.9$ & $-28.4 \pm 2.6$ \\
\hline EE-CDDP (\%) & $86.1 \pm 2.9$ & $85.4 \pm 3.3$ & NA & NA & $84.9 \pm 2.3$ \\
\hline EE-DOX (\%) & $82.3 \pm 3.1$ & NA & $83.4 \pm 2.8$ & NA & $82.7 \pm 2.6$ \\
\hline DL-CDDP (\%) & $3.8 \pm 0.5$ & $3.6 \pm 0.4$ & NA & NA & $4.7 \pm 0.5$ \\
\hline DL-DOX (\%) & $2.2 \pm 0.3$ & NA & $2.3 \pm 0.3$ & NA & $3.2 \pm 0.3$ \\
\hline
\end{tabular}

EGF C/D LPNs, EGFR-targeted LPNs loaded with CDDP and DOX; EGF C LPNs, EGFR-targeted LPNs loaded with CDDP; EGF D LPNs, EGFR-targeted LPNs loaded with DOX; EGF LPNs, Drug-free EGFR-targeted LPNs; C/D LPNs, EGFR-free LPNs loaded with CDDP and DOX. CDDP, cisplatin; DOX, doxorubicin; EGFR, epidermal growth factor receptor; LPNs, lipid polymeric nanoparticles; PDI, polydispersity index; EE, encapsulation efficiency; DL, drug loading. N/A, not applicable.

Table II. $\mathrm{CI}_{50}$ values of EGF C/D LPNs.

\begin{tabular}{|c|c|c|c|c|}
\hline LPNs & CDDP/DOX (w/w) & $\mathrm{CI}_{50}$ of $\mathrm{CDDP}(\mathrm{mg} / \mathrm{ml})$ & $\mathrm{CI}_{50}$ of $\mathrm{DOX}(\mathrm{mg} / \mathrm{ml})$ & $\mathrm{CI}_{50}$ \\
\hline EGF C LPNs & NA & 9.26 & NA & NA \\
\hline EGF D LPNs & NA & NA & 11.37 & NA \\
\hline EGF C/D LPNs & $10: 1$ & 8.76 & 0.88 & 1.05 \\
\hline EGF C/D LPNs & $5: 1$ & 8.13 & 1.63 & 1.02 \\
\hline EGF C/D LPNs & $2: 1$ & 3.76 & 1.88 & 0.57 \\
\hline EGF C/D LPNs & $1: 1$ & 5.97 & 5.97 & 1.17 \\
\hline EGF C/D LPNs & $1: 2$ & 3.91 & 7.82 & 1.11 \\
\hline EGF C/D LPNs & $1: 5$ & 1.09 & 10.91 & 1.08 \\
\hline
\end{tabular}

EGF C/D LPNs, EGFR-targeted LPNs loaded with CDDP and DOX; EGF C LPNs, EGFR-targeted LPNs loaded with CDDP; EGF D LPNs, EGFR-targeted LPNs loaded with DOX. CDDP, cisplatin; DOX, doxorubicin; EGFR, epidermal growth factor receptor; LPNs, lipid polymeric nanoparticles; $\mathrm{CI}_{50}$, combination index calculated by the $\mathrm{IC}_{50}$ value of $\mathrm{CDDP}$ and DOX.
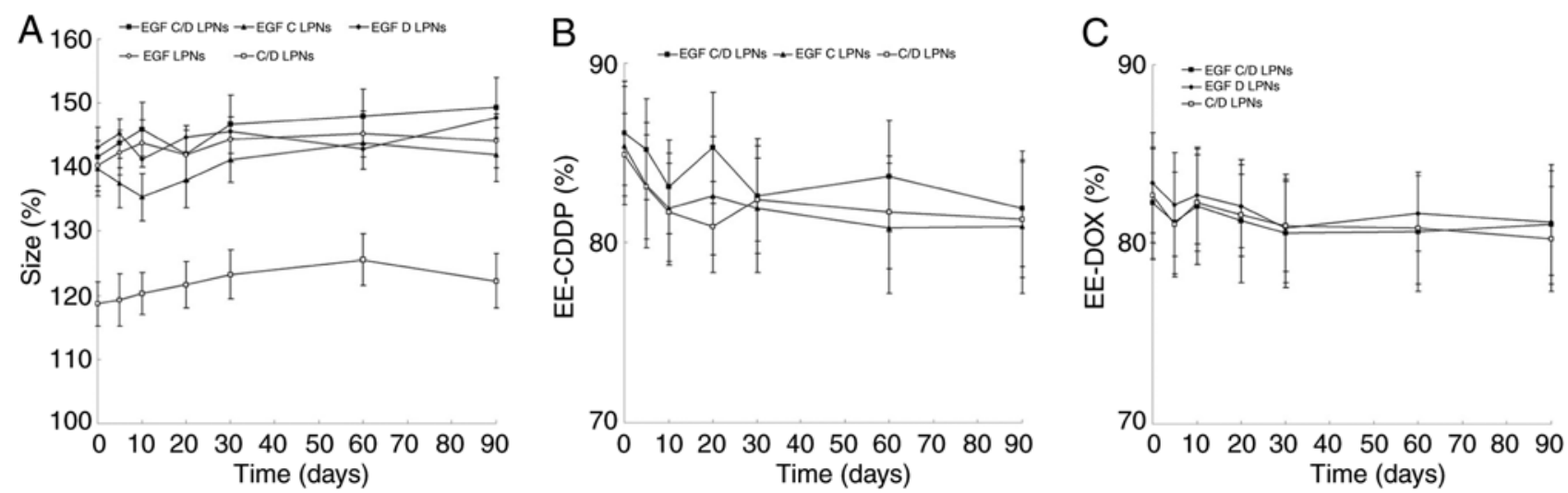

Figure 3. Stability of the LPNs was evaluated by the changes in size (A) and EE of CDDP (B) and DOX (C) over a period of 90 days. LPNs were stored at $2-8^{\circ} \mathrm{C}$. The size and EE LPNs were measured at $0,5,10,20,30,60$, and 90 days. Data are expressed as mean \pm standard deviation (n=6). EGF C/D LPNs, EGFR-targeted LPNs loaded with CDDP and DOX; EGF C LPNs, EGFR-targeted LPNs loaded with CDDP; EGF D LPNs, EGFR-targeted LPNs loaded with DOX; EGF LPNs, Drug-free EGFR-targeted LPNs; C/D LPNs, EGFR-free LPNs loaded with CDDP and DOX. CDDP, cisplatin; DOX, doxorubicin; EGFR, epidermal growth factor receptor; LPNs, lipid polymeric nanoparticles; EE, encapsulation efficiency.

end of the study could prove the efficiency of EGF modification. Tumor inhibition ratios of EGF C/D LPNs, EGF C LPNs and EGF D LPNs were 74.5, 45.6, and 49.7\%, respectively
(Fig. 7B). This revealed the superior antitumor ability of the dual drug LPNs than the single drug LPNs. The drug-loaded LPN groups caused a slightly increase in the weights of mice, 

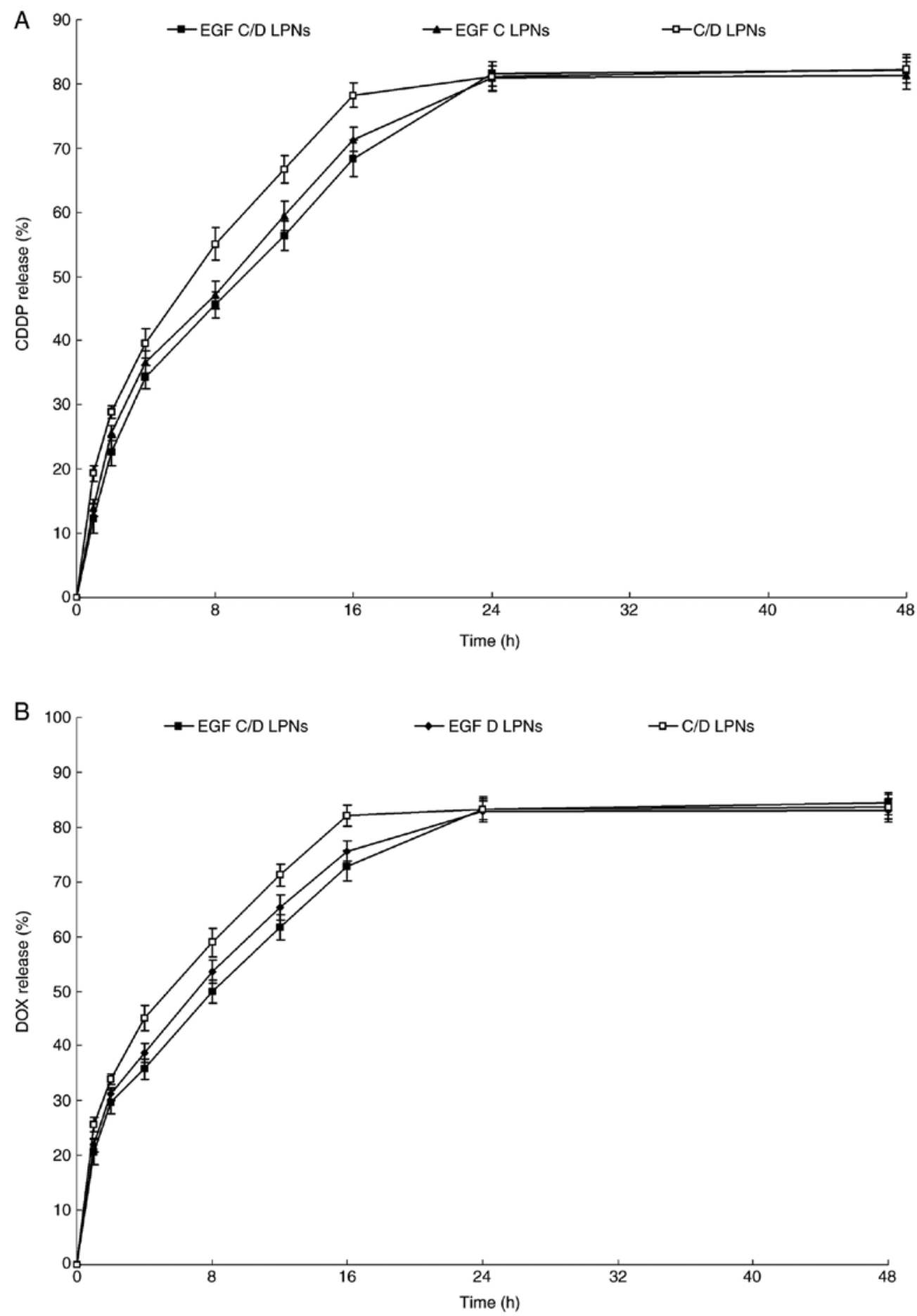

Figure 4. In vitro CDDP (A) and DOX (B) release profile of various types of LPNs. Drug release from LPNs was evaluated by dialysis method. Various types of LPNs $(2 \mathrm{ml})$ were sealed in dialysis bags (molecular cut-off: $1 \mathrm{kDa}$ ) separately, which were incubated in PBS $\left(\mathrm{pH} 7.4,37^{\circ} \mathrm{C}, 20 \mathrm{ml}\right)$ and shaken at the speed of $100 \mathrm{rpm}$. Data are expressed as mean \pm standard deviation $(\mathrm{n}=6)$. EGF C/D LPNs, EGFR-targeted LPNs loaded with CDDP and DOX; EGF C LPNs, EGFR-targeted LPNs loaded with CDDP; EGF D LPNs, EGFR-targeted LPNs loaded with DOX; C/D LPNs, EGFR-free LPNs loaded with CDDP and DOX. CDDP, cisplatin; DOX, doxorubicin; EGFR, epidermal growth factor receptor; LPNs, lipid polymeric nanoparticles.

while the body weights of the Free C/D, blank LPNs and saline control groups were significantly decreased (Fig. 7C).

\section{Discussion}

Epidermal growth factor receptor (EGFR) is overexpressed in many carcinomas and has been used as a promising target for drug delivery. For example, Singh et al developed
EGFR-targeted gelatin nanoparticles for systemic administration of gemcitabine in an orthotopic pancreatic cancer model. They synthesized a thiolated gelatin conjugate which they used for the loading of gemcitabine (34). Gill and colleagues reported the preparation of poly(lactic-co-glycolic acid) (PLGA) nanoparticles surface conjugated to diethylenetriaminepentaacetic acid-human EGF encapsulating the ruthenium-based DNA replication inhibitor and radiosensitizer 


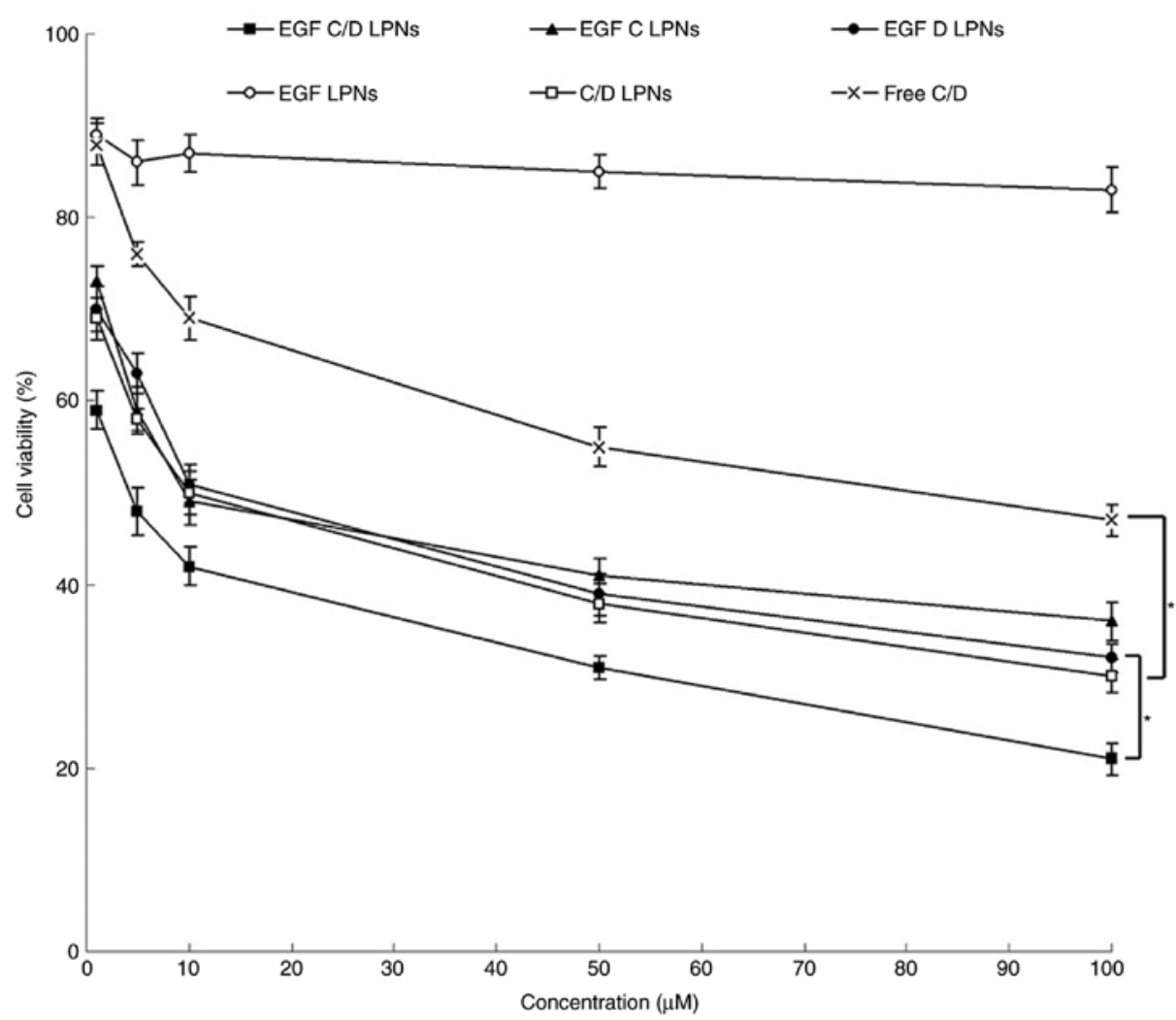

Figure 5. Cytotoxicity of LPNs evaluated using A549 cells by MTT assay. Cytotoxicity of LPNs was evaluated on A549 cells by MTT assay. Cells $\left(2 \times 10^{5}\right.$ per well) were seeded into 6-well microplates and allowed to grow for $24 \mathrm{~h}$ to a subconfluent state. Various types of LPNs and Free C/D were added along with fresh medium (contained 10\% of FBS) and incubated for $72 \mathrm{~h}$. Data are expressed as mean \pm standard deviation (n=8). "P<0.05. EGF C/D LPNs, EGFR-targeted LPNs loaded with CDDP and DOX; EGF C LPNs, EGFR-targeted LPNs loaded with CDDP; EGF D LPNs, EGFR-targeted LPNs loaded with DOX; EGF LPNs, Drug-free EGFR-targeted LPNs; C/D LPNs, EGFR-free LPNs loaded with CDDP and DOX; Free C/D, Free-CDDP and DOX drug combination. CDDP, cisplatin; DOX, doxorubicin; EGFR, epidermal growth factor receptor; LPNs, lipid polymeric nanoparticles.

A
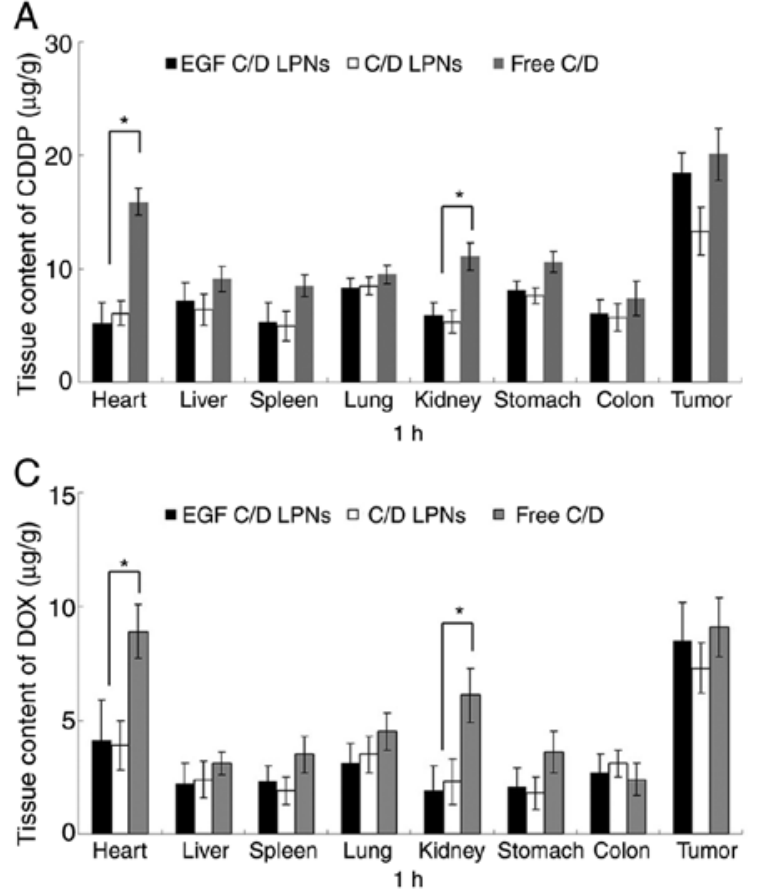
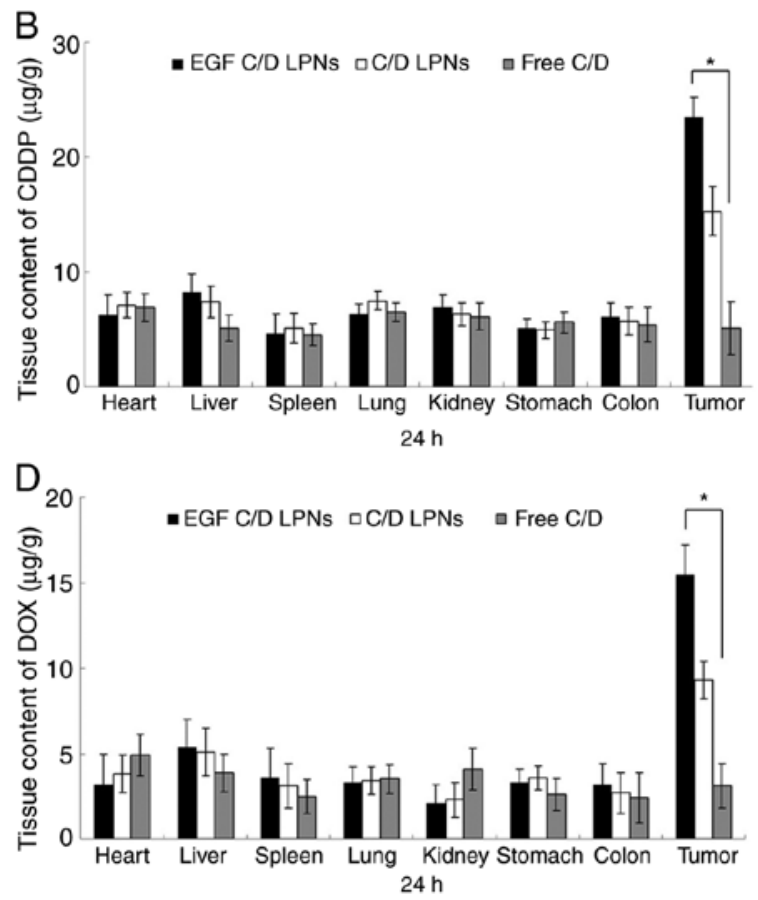

Figure 6. In vivo drug tissue distribution of EGF C/D LPNs, C/D LPNs and Free C/D in a lung carcinoma mouse model: CDDP distribution at 1 h (A) and $24 \mathrm{~h}(\mathrm{~B})$; DOX distribution at $1 \mathrm{~h}(\mathrm{C})$ and $24 \mathrm{~h}(\mathrm{D})$ The mice-bearing lung carcinoma model was randomly divided into several groups and intravenously administered (through the tail vein) $200 \mu \mathrm{l}$ of the various types of LPNs and Free C/D (each contained $5 \mathrm{mg}$ CDDP per $\mathrm{kg}$ and/or $2.5 \mathrm{mg}$ DOX per $\mathrm{kg}$ of mice), separately. Data are expressed as mean \pm standard deviation $(n=8)$. * $\mathrm{P}<0.05$. EGF C/D LPNs, EGFR-targeted LPNs loaded with CDDP and DOX; C/D LPNs, EGFR-free LPNs loaded with CDDP and DOX; Free C/D, Free-CDDP and DOX drug combination. CDDP, cisplatin; DOX, doxorubicin; EGFR, epidermal growth factor receptor; LPNs, lipid polymeric nanoparticles. 

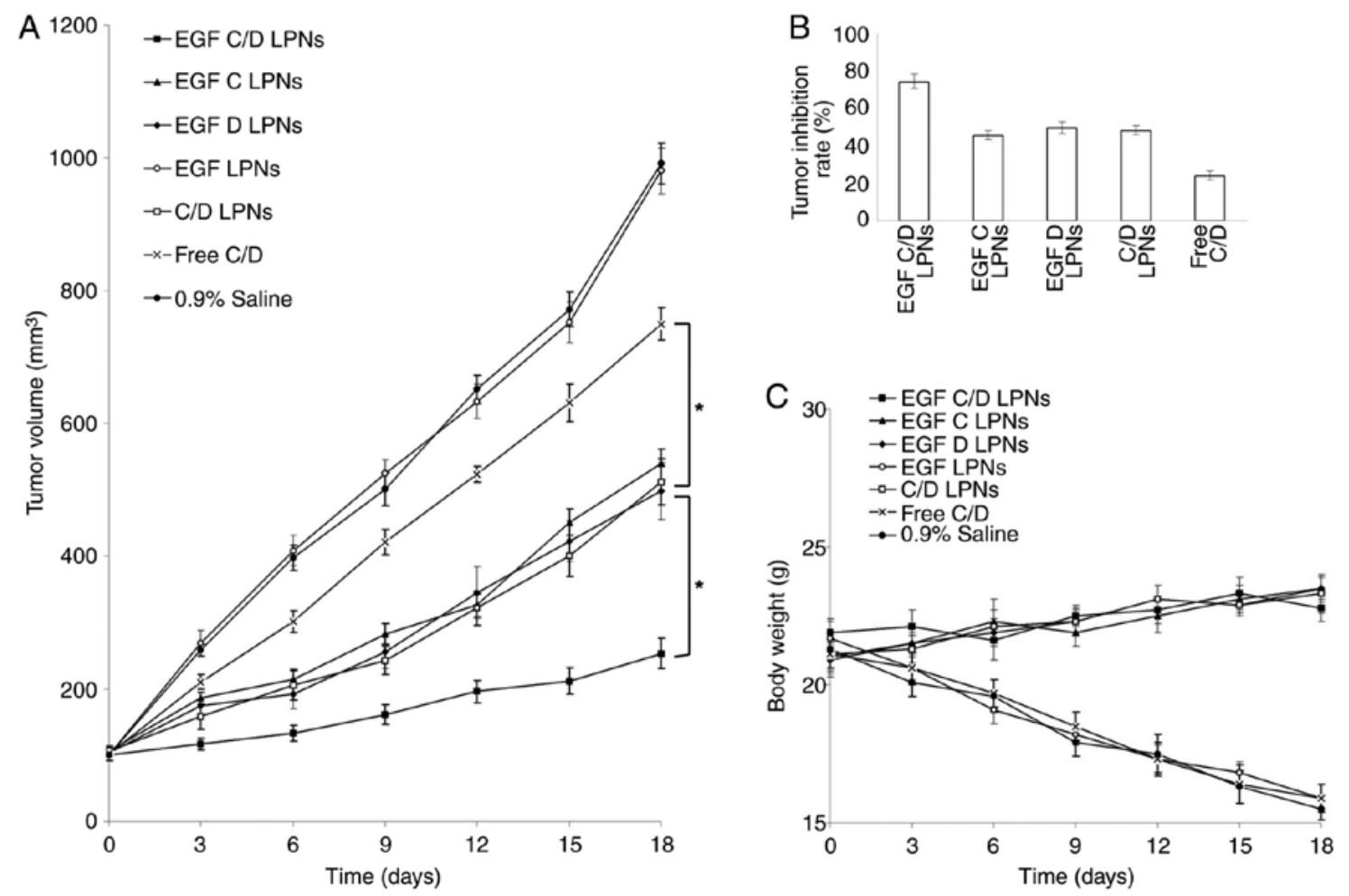

Figure 7. In vivo tumor inhibitory effect evaluated using a mouse-bearing lung carcinoma model: (A) Tumor volume, (B) tumor inhibition rate and (C) body weight. Data are expressed as mean \pm standard deviation $(\mathrm{n}=8)$. ${ }^{*} \mathrm{P}<0.05$. EGF C/D LPNs, EGFR-targeted LPNs loaded with CDDP and DOX; EGF C LPNs, EGFR-targeted LPNs loaded with CDDP; EGF D LPNs, EGFR-targeted LPNs loaded with DOX; EGF LPNs, Drug-free EGFR-targeted LPNs; C/D LPNs, EGFR-free LPNs loaded with CDDP and DOX; Free C/D, Free-CDDP and DOX drug combination. CDDP, cisplatin; DOX, doxorubicin; EGFR, epidermal growth factor receptor; LPNs, lipid polymeric nanoparticles.

for EGFR-targeted combination therapy in esophageal cancer cells (35). Kuai et al designed EGFR-targeted immune magnetic liposomes which could capture circulating colorectal tumor cells efficiently (36). In the present study, EGF-PEG-DSPE was synthesized and applied as materials for lipid polymeric nanoparticle (LPN) preparation. The core-shell structure of the LPNs allows them to achieve high drug encapsulation yield, excellent stability, tunable and sustained drug release profile, and potential for differential targeting of cells or tissues (37). The stability of the LPNs was essential to be evaluated since disruption of the particles could affect the therapeutic potential of the drug delivery systems (38). The sizes and encapsulation efficiency (EE) of all LPNs tested showed no obvious change during 90 days, indicating that the LPNs were stable during 3 months of storage without the incidence of disassembly or aggregation.

For the preparation of EGF C/D LPNs, cisplatin (CDDP) was encapsulated in the polymeric PLA core and doxorubicin (DOX) was beneath the lipid shell. The release of DOX from LPNs was faster than CDDP. This may be explained by the fact that the lipid shell on the surface of LPNs enabled DOX to be released faster than CDDP. Compared with C/D LPNs, drugs release from EGF C/D LPNs were in a more sustained behavior, which may be due to the modification of EGF that hindered the drug release from the systems. The prolongation of release time may be attributed to the slow degradation of the nanomaterials, which let the drugs slowly diffuse from the matrix (39).

Cytotoxicity of LPNs was tested in A549 cells. The retained cytotoxicity of the LPNs in a long therapeutic period is important for treatment (40). The results showed that there was no significant cytotoxicity of drug-free EGF LPNs, which may be proof of the low toxicity of the materials used in the preparation. Significant improvement in cytotoxicity was achieved by the drug-loaded LPNs when compared to the free drugs. EGFC/DLPNs inhibited the growth of lung carcinoma cells more significantly compared with that noted in the C/D LPNs, which indicated that EGF modification enhanced the cytotoxicity of the system (41). Combination index (CI) was calculated to validate the synergistic effect of the drug co-loaded LPNs using the isobologram equation of Chou and Talalay (42). EGF C/D LPNs displayed a CI value $<1(0.57)$ when the $\mathrm{CDDP} / \mathrm{DOX}$ ratio was 2:1, suggesting the suitable CDDP to DOX ratio in the LPNs system. However, other ratios showed no synergistic effect than the single drug-loaded LPNs. The reason might be attributed to differences in the controlled release manner and also different dosages of drugs have different effects on cancer cells $(24,43)$. The drug-loaded particles were mainly taken up by cells via the endocytosis pathway and then exerted antitumor activity after the drug molecules were released from the NPs, thus different dosages and ratios of drugs may have different effects, some synergistic and others antagonistic.

In vivo tissue biodistribution behavior of EGF C/D LPNs, C/D LPNs and Free C/D were evaluated in a lung carcinoma mouse model. At $24 \mathrm{~h}$ after injection, tumor tissue showed a significantly higher accumulation of EGF C/D LPNs than those in other normal tissues, which supported the preferential accumulation of EGF C/D LPNs in the tumor based on the enhanced permeability and retention (EPR) effect (44). The long 
circulating effect of LPNs was attributed to the presence of a PEG chain on the surface of the particles, which provided stealth effect to the NLCs (45). Less CDDP and DOX containing LPNs accumulated in the heart and kidney than Free C/D at $1 \mathrm{~h}$ after injection; this may lead to lower toxicity in the heart and kidney.

In vivo antitumor efficiency of EGF C/D LPNs was more prominently than C/D LPNs and the single drug-loaded LPNs. This may be attributed to the synergistic anti-lung carcinoma effects of the dual drug-loaded LPNs. C/D LPNs exhibited more profound efficiency than the Free $\mathrm{C} / \mathrm{D}$, which could be explained by the high structural integrity, high biocompatibility and bioavailability and controlled release capability attributed to the polymer core of the lipid layers of the LPNs (46). The lipid shell enveloping the core is biocompatible and exhibits behavior similar to that of cell membranes. Thus, LPNs have good affinity to the cell membranes, allow fusion of the particles to the cell surface and drugs are able to be delivered more efficiently into tumor cells (47). Considering the lower toxicity of LPNs due to the body weight loss of the animals, EGF C/D LPNs exhibited improved anticancer activity along with lower toxicity than the free drugs. The tumor inhibition ratio of the EGF C/D LPNs was significantly higher than that of the EGF C LPNs, EGF D LPNs and C/D LPNs. These results are in accordance with the above results, indicating a superior efficiency of EGF C/D LPNs for lung carcinoma therapy.

In conclusion, EGF-PEG-DSPE was synthesized and EGFR-targeted LPNs were constructed, which consisted of a CDDP-loaded hydrophobic polymeric core, DOX-loaded phospholipid layer, and an outer layer of EGF-PEG-DSPE ligand. EGF C/D LPNs were stable and could release drugs in a sustained manner. In vitro and in vivo studies revealed that the EGF C/D LPNs exhibited improved anticancer activity along with lower toxicity. These results indicated superior efficiency of the EGF C/D LPNs for lung carcinoma therapy.

\section{Acknowledgements}

Not applicable.

\section{Funding}

No funding was received.

\section{Availability of data and materials}

The datasets used during the present study are available from the corresponding author upon reasonable request.

\section{Authors' contributions}

YN designed the research, performed the experiments and analyzed the data. YN wrote the paper and approved the manuscript and agrees to be accountable for all aspects of the research in ensuring that the accuracy or integrity of any part of the work are appropriately investigated and resolved.

\section{Ethics approval and consent to participate}

All animal experiments were approved by the Ethics Committee of the Affiliated Hospital of Hebei University and followed the SUNY Upstate Medical University, Department of Laboratory Animal Resources Guidelines for Anesthesia and Analgesia in Laboratory Animals and Guidelines for the Care and Use of Laboratory Animals and the National Animal Laboratory Center of China.

\section{Patient consent for publication}

Not applicable.

\section{Competing interests}

The authors state that they have no competing interests.

\section{References}

1. Simonsen DF, Farkas DK, Søgaard M, Horsburgh CR, Sørensen HT and Thomsen RW: Tuberculosis and risk of cancer: A Danish nationwide cohort study. Int J Tuberc Lung Dis 18: 1211-1219, 2014

2. Siegel RL, Miller KD and Jemal A: Cancer statistics, 2017. CA Cancer J Clin 67: 7-30, 2017.

3. Song Z, Shi Y, Han Q and Dai G: Endothelial growth factor receptor-targeted and reactive oxygen species-responsive lung cancer therapy by docetaxel and resveratrol encapsulated lipid-polymer hybrid nanoparticles. Biomed Pharmacother 105: 18-26, 2018.

4. Carney DN: Lung cancer-time to move on from chemotherapy. N Engl J Med 346: 126-128, 2002.

5. Reck M,Heigener DF, Mok T, Soria JC and Rabe KF: Management of non-small-cell lung cancer: Recent developments. Lancet 382: 709-719, 2013.

6. Murren JR, Durivage HJ, Rosenberg AH, Chen Y, Del Prete SA, Murphy GJ, Buzaid AC and Hait WN: Cisplatin, doxorubicin, mitomycin $\mathrm{C}$, and 5-fluorouracil for the treatment of metastatic non-small cell lung cancer. Limited activity of an aggressive chemotherapy regimen. Am J Clin Oncol 17: 239-241, 1994

7. Li Z, Song M, He Z, Zong L, Jiang B, Zhang T and Hu Z: Comparison of quick recovery outcome of inhalable doxorubicin and cisplatin in lung cancer patients: A randomized, double-blind, single-center trial. Drug Deliv Transl Res 8: 985-993, 2018.

8. Liu J, Cheng H, Han L, Qiang Z, Zhang X, Gao W, Zhao K and Song Y: Synergistic combination therapy of lung cancer using paclitaxel- and triptolide-coloaded lipid-polymer hybrid nanoparticles. Drug Des Devel Ther 12: 3199-3209, 2018.

9. Prabhu VV and Devaraj N: Epidermal growth factor receptor tyrosine kinase: A potential target in treatment of non-small-cell lung carcinoma. J Environ Pathol Toxicol Oncol 36: 151-158, 2017.

10. Pancewicz-Wojtkiewicz J: Epidermal growth factor receptor and notch signaling in non-small-cell lung cancer. Cancer Med 5: 3572-3578, 2016.

11. Schrank Z, Chhabra G, Lin L, Iderzorig T, Osude C, Khan N, Kuckovic A, Singh S, Miller RJ and Puri N: Current molecular-targeted therapies in NSCLC and Their mechanism of resistance. Cancers (Basel) 10: pii: E224, 2018.

12. Mendelsohn $\mathrm{J}$ and Baselga $\mathrm{J}$ : Epidermal growth factor receptor targeting in cancer. Semin Oncol 33: 369-385, 2006.

13. Gerber DE: Targeted therapies: A new generation of cancer treatments. Am Fam Physician 77: 311-319, 2008.

14. Tsai WH, Yu KH, Huang YC and Lee CI: EGFR-targeted photodynamic therapy by curcumin-encapsulated chitosan/TPP nanoparticles. Int J Nanomedicine 13: 903-916, 2018.

15. Iram S, Zahera M, Khan S, Khan I, Syed A, Ansary AA, Ameen F, Shair OHM and Khan MS: Gold nanoconjugates reinforce the potency of conjugated cisplatin and doxorubicin. Colloids Surf B Biointerfaces 160: 254-264, 2017.

16. Sun TM, Du JZ, Yao YD, Mao CQ, Dou S, Huang SY, Zhang PZ, Leong KW, Song EW and Wang J: Simultaneous delivery of siRNA and paclitaxel via a 'two-in-one' micelleplex promotes synergistic tumor suppression. ACS Nano 5: 1483-1494, 2011.

17. Browning RJ, Reardon PJT, Parhizkar M, Pedley RB, Edirisinghe M, Knowles JC and Stride E: Drug delivery strategies for platinum-based chemotherapy. ACS Nano 11: 8560-8578, 2017. 
18. Oberoi HS, Nukolova NV, Kabanov AV and Bronich TK: Nanocarriers for delivery of platinum anticancer drugs. Adv Drug Deliv Rev 65: 1667-1685, 2013.

19. Marullo R, Werner E, Degtyareva N, Moore B, Altavilla G, Ramalingam SS and Doetsch PW: Cisplatin induces a mitochondrial-ROS response that contributes to cytotoxicity depending on mitochondrial redox status and bioenergetic functions. PLoS One 8: e81162, 2013.

20. Ramasamy T, Tran TH, Choi JY, Cho HJ, Kim JH, Yong CS, Choi HG and Kim JO: Layer-by-layer coated lipid-polymer hybrid nanoparticles designed for use in anticancer drug delivery. Carbohydr Polym 102: 653-661, 2014.

21. Ruttala HB, Ramasamy T, Gupta B, Choi HG, Yong CS and Kim JO: Multiple polysaccharide-drug complex-loaded liposomes: A unique strategy in drug loading and cancer targeting. Carbohydr Polym 173: 57-66, 2017.

22. Guo S, Wang Y, Miao L, Xu Z, Lin CM, Zhang Y and Huang L: Lipid-coated Cisplatin nanoparticles induce neighboring effect and exhibit enhanced anticancer efficacy. ACS Nano 7: 9896-9904, 2013.

23. Liu B, Han L, Liu J, Han S, Chen Z and Jiang L: Co-delivery of paclitaxel and TOS-cisplatin via TAT-targeted solid lipid nanoparticles with synergistic antitumor activity against cervical cancer. Int J Nanomedicine 12: 955-968, 2017.

24. Kou CH, Han J, Han XL, Zhuang HJ and Zhao ZM: Preparation and characterization of the Adriamycin-loaded amphiphilic chitosan nanoparticles and their application in the treatment of liver cancer. Oncol Lett 14: 7833-7841, 2017.

25. Bohl Kullberg E, Bergstrand N, Carlsson J, Edwards K, Johnsson M, Sjöberg S and Gedda L: Development of EGF-conjugated liposomes for targeted delivery of boronated DNA-binding agents. Bioconjug Chem 13: 737-743, 2002.

26. Qiu J, Cai G, Liu X and Ma D: $\alpha_{\mathrm{v}} \beta_{3}$ integrin receptor specific peptide modified, salvianolic acid $B$ and panax notoginsenoside loaded nanomedicine for the combination therapy of acute myocardial ischemia. Biomed Pharmacother 96: 1418-1426, 2017

27. Zhang Y, Zhang $\mathrm{P}$ and Zhu T: Ovarian carcinoma biological nanotherapy: Comparison of the advantages and drawbacks of lipid, polymeric, and hybrid nanoparticles for cisplatin delivery. Biomed Pharmacother 109: 475-483, 2019.

28. Tan S and Wang G: Redox-responsive and $\mathrm{pH}$-sensitive nanoparticles enhanced stability and anticancer ability of erlotinib to treat lung cancer in vivo. Drug Des Devel Ther 11: 3519-3529, 2017.

29. Tan S and Wang G: Lung cancer targeted therapy: Folate and transferrin dual targeted, glutathione responsive nanocarriers for the delivery of cisplatin. Biomed Pharmacother 102: 55-63, 2018

30. Duan W and Liu Y: Targeted and synergistic therapy for hepatocellular carcinoma: Monosaccharide modified lipid nanoparticles for the co-delivery of doxorubicin and sorafenib. Drug Des Devel Ther 12: 2149-2161, 2018.

31. Yang F, Li A, Liu H and Zhang H: Gastric cancer combination therapy: Synthesis of a hyaluronic acid and cisplatin containing lipid prodrug coloaded with sorafenib in a nanoparticulate system to exhibit enhanced anticancer efficacy and reduced toxicity. Drug Des Devel Ther 12: 3321-3333, 2018.

32. Wang Z, Wei Y, Fang G, Hong D, An L, Jiao T, Shi Y and Zang A: Colorectal cancer combination therapy using drug and gene co-delivered, targeted poly(ethylene glycol)- $\varepsilon$-poly(caprolactone) nanocarriers. Drug Des Devel Ther 12: 3171-3180, 2018.

33. Gao Z, Li Z, Yan J and Wang P: Irinotecan and 5-fluorouracil-co-loaded, hyaluronic acid-modified layer-by-layer nanoparticles for targeted gastric carcinoma therapy. Drug Des Devel Ther 11: 2595-2604, 2017.
34. Singh A, Xu J, Mattheolabakis G and Amiji M: EGFR-targeted gelatin nanoparticles for systemic administration of gemcitabine in an orthotopic pancreatic cancer model. Nanomedicine 12: 589-600, 2016

35. Gill MR, Menon JU, Jarman PJ, Owen J, Skaripa-Koukelli I, Able S, Thomas JA, Carlisle R and Vallis KA: ${ }^{111}$ In-labelled polymeric nanoparticles incorporating a ruthenium-based radiosensitizer for EGFR-targeted combination therapy in oesophageal cancer cells. Nanoscale 10: 10596-10608, 2018

36. Kuai JH, Wang Q, Zhang AJ, Zhang JY, Chen ZF, Wu KK and $\mathrm{Hu} \mathrm{XZ}$ : Epidermal growth factor receptor-targeted immune magnetic liposomes capture circulating colorectal tumor cells efficiently. World J Gastroenterol 24: 351-359, 2018.

37. Zhang L, Chan JM, Gu FX, Rhee JW, Wang AZ, Radovic-Moreno AF, Alexis F, Langer R and Farokhzad OC: Self-assembled lipid-polymer hybrid nanoparticles: A robust drug delivery platform. ACS Nano 2: 1696-1702, 2008.

38. You P, Yuan R and Chen C: Design and evaluation of lidocaineand prilocaine-coloaded nanoparticulate drug delivery systems for topical anesthetic analgesic therapy: A comparison between solid lipid nanoparticles and nanostructured lipid carriers. Drug Des Devel Ther 11: 2743-2752, 2017.

39. Zhang S, Li J, Hu S, Wu F and Zhang X: Triphenylphosphonium and D- $\alpha$-tocopheryl polyethylene glycol 1000 succinate-modified, tanshinone IIA-loaded lipid-polymeric nanocarriers for the targeted therapy of myocardial infarction. Int J Nanomedicine 13: 4045-4057, 2018.

40. Miao JF, Peng YF, Chen S, Gao WJ, Yang QX, Zhu P, Guo J, Tao J, Luo L, Zhang Y and Ling Y: A novel harmine derivative, N-(4-(hydroxycarbamoyl)benzyl)-1-(4-methoxyphenyl)-9H-pyrido[3,4-b]indole-3-carboxamide (HBC), as histone deacetylase inhibitor: In vitro antiproliferation, apoptosis induction, cell cycle arrest, and antimetastatic effects. Eur J Pharmacol 824: 78-88, 2018.

41. Nishikawa K, Asai T, Shigematsu H, Shimizu K, Kato H, Asano Y, Takashima S, Mekada E, Oku N and Minamino T: Development of anti-HB-EGF immunoliposomes for the treatment of breast cancer. J Control Release 160: 274-280, 2012.

42. Chou TC and Talalay P: Quantitative analysis of dose-effect relationships: The combined effects of multiple drugs or enzyme inhibitors. Adv Enzyme Regul 22: 27-55, 1984

43. Gawde KA, Sau S, Tatiparti K, Kashaw SK, Mehrmohammadi M, Azmi AS and Iyer AK: Paclitaxel and di-fluorinated curcumin loaded in albumin nanoparticles for targeted synergistic combination therapy of ovarian and cervical cancers. Colloids Surf B Biointerfaces 167: 8-19, 2018

44. Wang H, Sun G, Zhang Z and Ou Y: Transcription activator, hyaluronic acid and tocopheryl succinate multi-functionalized novel lipid carriers encapsulating etoposide for lymphoma therapy. Biomed Pharmacother 91: 241-250, 2017.

45. Cui T, Zhang S and Sun H: Co-delivery of doxorubicin and $\mathrm{pH}$-sensitive curcumin prodrug by transferrin-targeted nanoparticles for breast cancer treatment. Oncol Rep 37: 1253-1260, 2017.

46. Zhu B, Yu L and Yue Q: Co-delivery of vincristine and quercetin by nanocarriers for lymphoma combination chemotherapy. Biomed Pharmacother 91: 287-294, 2017.

47. Mandal B, Bhattacharjee H, Mittal N, Sah H, Balabathula P, Thoma LA and Wood GC: Core-shell-type lipid-polymer hybrid nanoparticles as a drug delivery platform. Nanomedicine 9: 474-491, 2013. 